\title{
Risk Management Practices at University Clinics in Communication Sciences and Disorders
}

\author{
Dorothy Neave-DiToro \\ Brooklyn College, City University of New York, dditoro@brooklyn.cuny.edu \\ Donald A. Vogel \\ Hunter College, City University of New York, dvoge@hunter.cuny.edu \\ Susan E. Wortsman \\ Hunter College, City University of New York, swortsma@hunter.cuny.edu
}

See next page for additional authors

DOI: doi.org/10.30707/TLCSD2.2Neave-DiToro

Follow this and additional works at: https://ir.library.illinoisstate.edu/tlcsd

Part of the Communication Commons

\section{Recommended Citation}

Neave-DiToro, Dorothy; Vogel, Donald A.; Wortsman, Susan E.; and Cascella, Paul W. (2018) "Risk Management Practices at University Clinics in Communication Sciences and Disorders," Teaching and Learning in Communication Sciences \& Disorders: Vol. 2: Iss. 2, Article 3.

DOI: doi.org/10.30707/TLCSD2.2Neave-DiToro

Available at: https://ir.library.illinoisstate.edu/tlcsd/vol2/iss2/3

This Pilot Studies is brought to you for free and open access by ISU ReD: Research and eData. It has been accepted for inclusion in Teaching and Learning in Communication Sciences \& Disorders by an authorized editor of ISU ReD: Research and eData. For more information, please contact ISUReD@ilstu.edu. 


\title{
Risk Management Practices at University Clinics in Communication Sciences and Disorders
}

\begin{abstract}
This study surveyed university clinic directors of audiology and speech-language pathology programs throughout the United States, and identified the content and risk management (RM) practices embedded into graduate curricula. One hundred and two (102) respondents provided information, via an electronic survey, about their campus and departmental RM practices. More than half of the programs reported embedding RM concepts into at least one graduate course yet only slightly above half of the programs assessed students' RM knowledge. Pre-professional students were most often trained in protecting patient's privacy and fire drills. Approximately one-third participated in university-wide programs, and 11 of the 102 programs reported a freestanding RM committee. In contrast, the clinic directors reported frequent RM monitoring activities, including yearly updates to policy and procedure manuals, equipment and documentation audits, and monitoring of fire drill and in-service attendance records. University clinics are only somewhat invested in RM topics and pedagogy. Thus, pre-professional students may not be fully informed about RM in work settings. University clinics, American Speech-Language-Hearing Association and the American Academy of Audiology are encouraged to further evaluate the content and breadth of candidates' knowledge and experiences to ensure that RM coursework and resources address this contemporary and critical topic.
\end{abstract}

\section{Keywords}

audiology, risk management, speech-language pathology

\section{Cover Page Footnote}

Acknowledgements: The authors would like to acknowledge and thank Michael Bergen for his input in the preparation of the manuscript. They would also like to thank the peer reviewers for their suggested revisions and comments on a previous draft of this paper.

\section{Authors}

Dorothy Neave-DiToro, Donald A. Vogel, Susan E. Wortsman, and Paul W. Cascella 
The American Speech-Language-Hearing Association (ASHA) (2016) and the American Academy of Audiology (AAA) (2016) advocate for risk management (RM) in work settings, emphasizing the implementation of practice standards to improve patient outcomes, promote safe work environments, and reduce practitioner financial loss and legal liability. The origins of the term RM can be traced to the early and middle parts of the twentieth century when it was embraced by the financial and business communities, as well as hospitals, medical professionals, and healthcare organizations (The Joint Commission, 2016; Dionne, 2013). Emphasis on RM has continued with the installation of the not-for-profit organization, The Joint Commission, and in the latter part of the century, with the founding of the American Society for Healthcare Risk Management (ASHRM, n.d.). Streimelweger, Wac, and Seiringer (2015) further discussed RM as a mechanism to assess and mitigate potential errors and adverse effects in healthcare settings.

In the same vein, the World Health Organization (WHO) (2011) has highlighted the need for training in patient safety at the undergraduate and graduate level for students in healthcare fields, and has classified it as a worldwide issue. In 2011, a curriculum guide on patient safety was released by the WHO, and in 2015, the WHO reported findings of a field test of university administrators, faculty and students based on the implementation of the WHO Multi-Professional Patient Safety Curriculum Guide (Farley, Zheng, Rousi \& Leotsakos, 2015). The field test included twelve universities, representing worldwide geographical regions, cultures and various economic backgrounds. The organization reported that the patient safety guide was written to be used by staff through academic faculty, and that the guidelines are culturally sensitive and written in easy to understand language. Overall, the feedback on the guide was positive and recommendations based on the field tests were that academic institutions could adapt the WHO patient safety guidelines based on the discipline and locality.

Enacting policies to ensure the safety of and benefits to patients and protection against financial loss and legal liability are important aspects for healthcare professionals to address. In the United States, while the highest rates of malpractice claims are waged against physicians (Bal, 2009), allied healthcare professionals such as physical therapists (Kolber \& Lucado, 2005) and those working in the communication sciences and disorders (CSD) fields (Lubinski \& Hudson, 2013) also face the risk of negligence lawsuits. Despite these risks, a recent survey of 437 allied health professionals found inconsistency in how RM was implemented despite RM advocacy by healthcare organizations (Leggat et al., 2016). Hopkin (2017) discussed how, with planning, identified risks in the workplace can be controlled. Lubinski \& Hudson (2013) discuss that liability is a topic that affects CSD professionals in all work environments and needs to be considered in terms of documentation of all patient interactions. The CSD field could look to other professions, such as nursing and physical therapy, to develop sound RM practices for clinical training (Johnstone \& Kanitsaki, 2007; Johnstone \& Kanitsaki, 2008; Kolber \& Lucado, 2005; Vincent et al, 2000).

RM is not only valued in ASHA and AAA professional settings, but is also salient to graduate curricula as evidenced in requirements set forth by the Council for Clinical Certification in Audiology and Speech-Language Pathology (CFCC), the Council on Academic Accreditation in Audiology and Speech-Language Pathology (CAA), and the Accreditation Commission for Audiology Education (ACAE) (ACAE, 2016; CAA, 2016; CFCC, 2012). The CAA and ACAE standards, for example, require graduate programs to train pre-professionals in Health Insurance Portability and Accountability Act (HIPAA), Family Educational Rights and Privacy Act 
(FERPA), and universal precautions (ACAE, 2016; CAA, 2016). These standards are regularly updated to meet the demands within the audiology (AUD) and speech-language pathology (SLP) fields. According to ASHA (1994), RM is akin to Quality Improvement initiatives, and RM programs include, (a) identifying potential risks, (b) analyzing the degree of risk, (c) developing and implementing risk control techniques, (d) monitoring RM effectiveness, and (e) professional RM education. As well, members of both associations are ethically mandated to protect the safety and welfare of clients (AAA, 2016; ASHA, 2016).

In addition to teaching students to be knowledgeable about the risks they face, it is important that graduate clinics have measures in place to minimize legal risks for the university and university employees, and to maintain the program's good standing and integrity. Gilfoyle (2008) discusses from a legal standpoint best practices for professional psychology training programs when addressing student competencies in clinical internships/externships. The article highlights possible legal ramifications related to encounters with students that lack the essential functions/requirements necessary for clinical work and/or students that are considered to be a possible direct threat to those around them. While the Guilfoyle (2008) article was not written to specifically address CSD professional training, many of the issues, legal matters and suggestions could be applied. For example, within the CSD profession, both the Council of Academic Programs in Communication Sciences and Disorders (CAPCSD) (2007) and ASHA (2007) have highlighted the essential functions required of CSD students.

Johnstone and Kanitsaki (2008) discusses that RM in healthcare is a worldwide issue that needs to be addressed and calls for further research in this area. While ASHA and AAA have existing RM standards, there are no professional reports describing how RM elements are addressed or assessed in academic training programs for AUD and SLP. Due to the important nature of this topic, and the limited information available on this topic in the CSD field, this research surveyed university clinic directors about RM practices at their CAA approved programs in AUD and SLP. The purpose was to obtain preliminary information about institutional and departmental RM committees, and RM pedagogy and content. This information is useful so that pre-professional training programs can understand the current state of clinical RM activities and to inform the development of RM guidelines for clinical training programs.

\section{Methods}

Survey Development. The authors constructed a RM survey that included a total of 29 questions (yes/no/not sure, multiple choice, scaled and open-ended) (see Appendix). Twenty questions were related to the RM practices within university CSD programs, three questions were to obtain demographic information on the individual completing the survey (number of years in clinic director role, number of years in CSD field, and area(s) of certification), and six questions were to obtain information about their programs (state where university is located, student populationundergraduates/graduates, services offered, number of clients seen per week, number of personnel in clinic, and number of students observing per week). The inclusion of various RM tasks was based on the literature search described below and the clinical experience of the authors. The survey was developed in four stages to further enhance its validity and reliability (Brancato et al, 2006). First, a draft survey was created based on a comprehensive literature review from 1994 to 2013 using electronic archival resources from ASHA, AAA, and publicly available databases including CINAHL, PUBMED, EBSCO, MEDLINE, and ERIC. Combinations of search terms 
included risk management, audiology, audiologist, speech-language pathology, speech-language pathologist, speech therapist, healthcare, schools, quality assurance, risk, risk reduction, risk avoidance, risk prevention, and risk transfer. Then, twelve public and private university clinic directors from the Metropolitan New York Council of University Clinic Directors in Communication Sciences and Disorders (MNYCUCD) independently reviewed the draft survey. These directors held their respective positions for no less than 5 years.

After incorporating the clinic directors' content, wording, and length suggestions, three City University of New York (CUNY) AUD and SLP faculty members (each with 15+ years' experience as clinic directors) and one advanced doctoral student with research design experience, also independently reviewed the revised survey's content, readability, and organization. Then, an associate clinic director, also affiliated with the MNYCUCD, offered comments about the survey's readability and content, after completing the amended survey herself. The output for this paper was generated using Qualtrics software (2014).

Distribution. Via Qualtrics survey software (Qualtrics, 2014), an email invitation and an electronic survey link was sent to 280 university clinic directors throughout the United States. The surveys were confidential and the software monitored completion statistics (i.e., number of surveys and rate) and prevented participants from taking the survey more than once. Participants had 60 days to complete the survey and received two reminder notices. The CUNY Institutional Review Board approved this research.

Participants. The participants were self-identified university clinic directors whose email addresses were obtained from a 280-member CAPCSD list that included nearly every American AUD and SLP training program. Preceding the survey, participants confirmed their consent to participate.

One hundred and two surveys were returned, a 36.4\% response rate. The participants held national certification (Certificate of Clinical Competence) in SLP (79.6\%), AUD (16.3\%), or both disciplines $(4.1 \%)$. Information was obtained regarding their experience in the CSD field $(M=$ 25.5 years; $S D=9.1$; range 2 to 41 years) and varied duration in their current director roles $(M=$ 9.5 years; $S D=7.2$; range $=<1$ to 34 years). The participants worked in 31 states and the District of Columbia, and represented all regions (Northeast, Southwest, West, Southeast, and Midwest) of the United States. Descriptive information about their university clinics is provided in table 1.

Table 1. Descriptive Information

Characteristics of the Participants' University Clinics

Number of Students

Graduate SLP Student-Clinicians

Graduate AuD Student-Clinicians

Undergraduate Student Clinicians

Number of Weekly Sessions

Speech-language Therapy

Speech-language Diagnostic Sessions

Audiology Diagnostic Sessions
$M(S D) \quad$ Range

$32.0(15.9) \quad 0$ to 80

$8.9(9.9) \quad 0$ to 40

$9.9(13.9) \quad 0$ to 67

$68.4(41.3) \quad 5$ to 200

$4.9(5.8) \quad 0$ to 37

$14.2(14.7) \quad 0$ to 80 


\begin{tabular}{lcc} 
Hearing Screenings & $14.9(25.0)$ & 0 to 197 \\
Hearing Aid Services & $12.2(11.4)$ & 0 to 50 \\
\hline
\end{tabular}

SLP = Speech-Language Pathology; AuD = Doctor of Audiology

\section{Results}

Institutional and Departmental RM Committees. A majority (64.7\%) of respondents reported having an institution-wide environmental health and safety program and 35.3\% of their clinics were included in the centralized program. In contrast, 25.5\% reported not knowing if a universitywide program existed, and $9.8 \%$ reported the absence of a university-wide program. A majority (89.2\%) reported the absence of a clinic-specific RM committee and the main reasons were: the clinic's inclusion in university RM activities (34.1\%), being unclear whether a RM committee was needed (28.6\%), a lack of prior consideration (21.1\%), a lack of RM knowledge (21.2\%), a lack of available time (20.9\%), and insufficient financial resources (10.9\%).

In contrast, 11 respondents $(10.8 \%)$ reported the presence of a departmental RM committee. Committee membership typically included the clinic director, clinical educators, administrative assistants, academic faculty, and graduate students. Six of the committees had funding from either the university, department or clinic budget. The frequency of departmental RM committee meetings ranged from monthly to once a semester to annually. Table 2 identifies the most and least frequently reported tasks of these committees. The tasks most frequently addressed privacy compliance, documentation monitoring via file audits, fire safety, patient privacy monitoring, and emergency preparedness.

Table 2. Risk Management Committee Tasks

\section{Most Frequently Reported RM-Related Tasks}

Compliance to Privacy Policies

Monitoring Client Documentation

Implementing Fire Safety Plans

Monitoring Patient Privacy

Emergency Preparedness

Verifying Credentialing Documentation

Implementing Infection Control Protocols

Least Frequently Reported RM-Related Tasks

Monitoring Chemicals Use/Implementation

Monitoring Equipment Calibration

Monitoring Patient Satisfaction

Monitoring Abuse Issues

Monitoring Affiliation Contracts

Training in Mobility and Transfers of Patients

Training Related to Asthma and Respiratory and Food Allergies

Clinic RM Activities and Content. A majority (62.8\%) of participants reported that RM was embedded into at least one course and $37.6 \%$ noted that $\mathrm{RM}$ was discussed during student meetings 
outside of regular classes. In contrast, $18.8 \%$ reported that RM was not a curriculum topic. A majority $(51.5 \%)$ reported that student RM knowledge was confirmed via an assessment tool, while $39.6 \%$ reported no RM assessment, and $7.0 \%$ reported a student self-assessment process. The participants indicated that student-clinicians were most often trained in HIPAA/privacy training, fire drills, and incidentally exposed to RM signage in their clinics (Figure 1). A majority $(86.1 \%)$ indicated HIPAA/privacy training for graduate student-clinicians and $48.5 \%$ for undergraduate students.

Figure 1.

Frequency of University Clinic RM Educational Activities

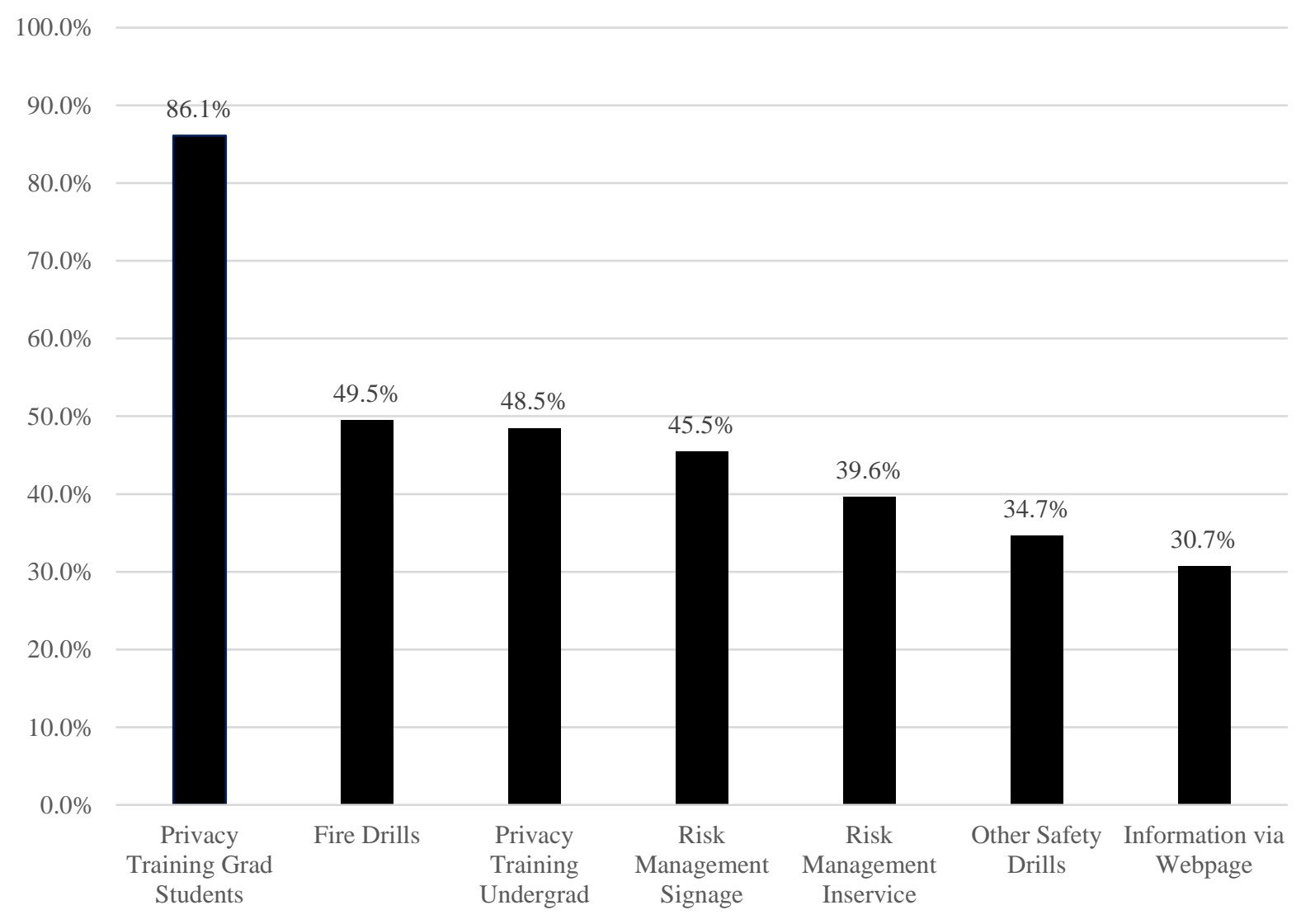

Clinic RM Efforts and Infractions. The participants reported frequent RM monitoring activities, including yearly updates to policy and procedure manuals, equipment and documentation audits, and monitoring of fire drill and in-service attendance records (See Figure 2). Participants also estimated the frequency of specific RM clinic incidents that were reported within the year. Sixtysix respondents $(64.7 \%)$ reported a cumulative total of 164 incidents across 12 incident types (See Figure 3). The most frequent incidents involved client documentation and/or breach of privacy issues, public safety problems, and externship contract issues. Some clinics reported having evacuation procedures, and six reported prior campus lockdowns. 
Teaching and Learning in Communication Sciences \& Disorders, Vol. 2 [2018], Iss. 2, Art. 3

Figure 2.

Frequency of RM Monitoring Activities at University Clinics

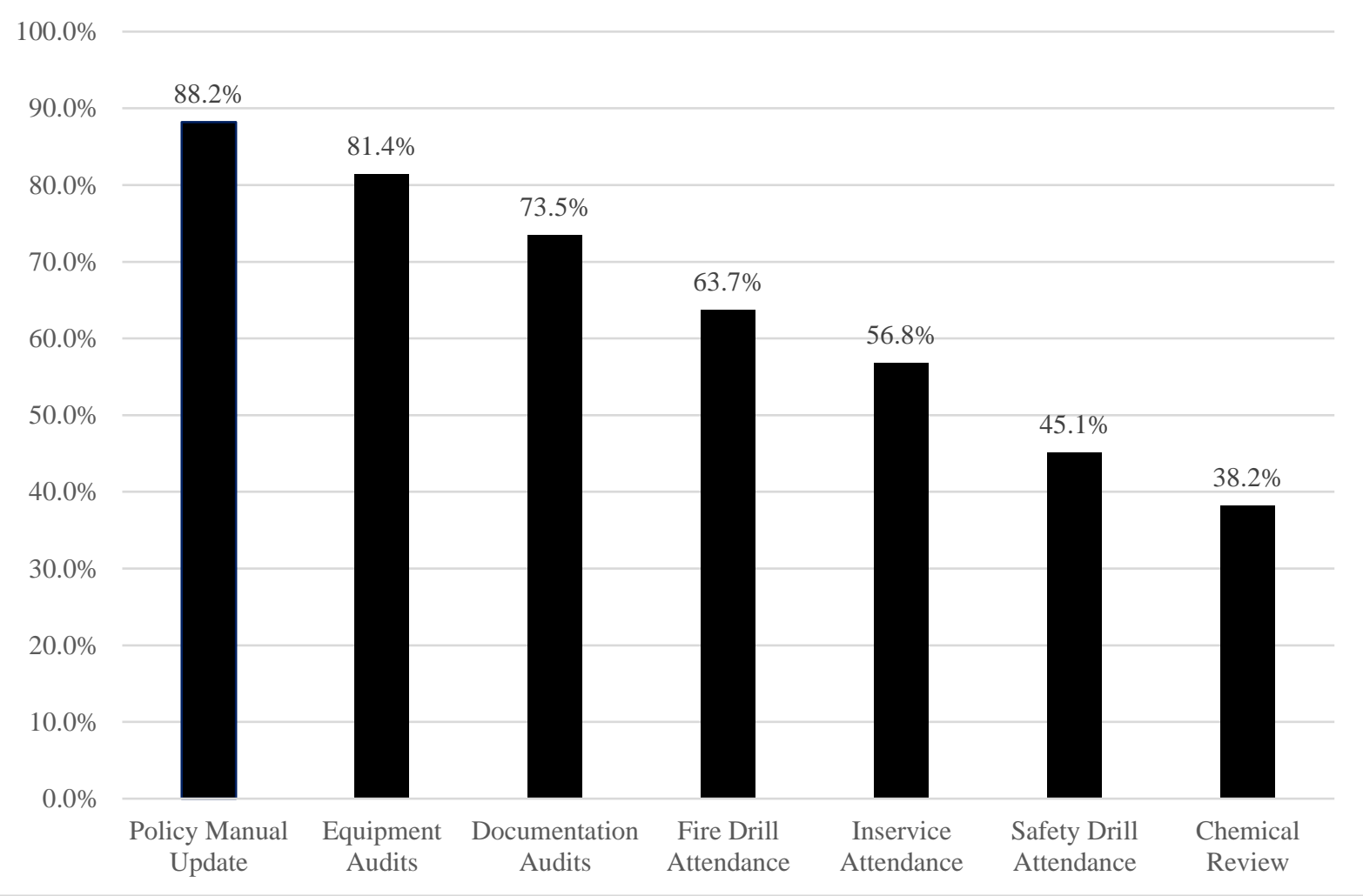

Figure 3.

University RM Clinic Infractions

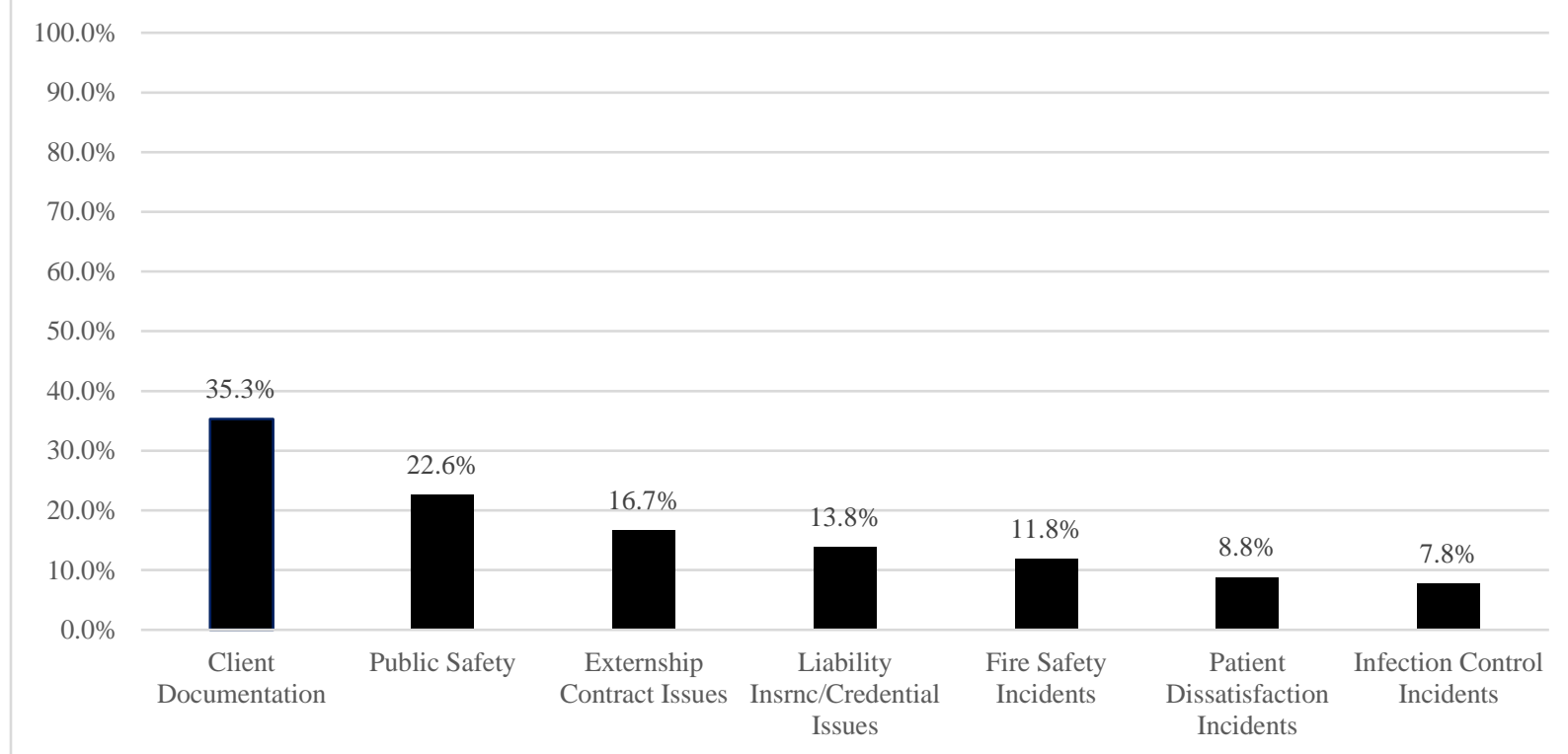




\section{Discussion}

Major Findings. Accredited AUD and SLP programs must demonstrate that pre-professional candidates are taught RM principles and activities, yet the emphasis on this important aspect of clinical training is not stressed and appears to not be clearly understood. Results of this survey revealed that slightly more than half of the programs addressed RM in at least one course, and 19 programs reported not including RM topics in the curriculum. Aspects of RM not being covered in the curriculum is highly unlikely and speaks to the premise of this article, that aspects of RM are not fully understood. For programs with RM activities, students participated in formal and informal activities whose content emphasized HIPAA and infection control training, fire safety, and being made aware of RM via clinic signage and program manuals. RM curricula did not appear to address abuse prevention, liability and externship contracts, patient satisfaction, public safety, or food/respiratory allergies. Slightly more than half of the programs assessed candidates' RM knowledge, and very few university clinics had their own RM committees, though some participated in campus RM activities. Programs with freestanding RM committees addressed health, safety, quality assurance, and prevention and educational tasks. Of the programs that reported to have a RM program, $16.83 \%$ reported that the legal department and/or administration utilized clinic documentation and $21.78 \%$ reported that having a RM program resulted in being appropriately prepared for incidents that occurred involving students.

Nearly all respondents reported monitoring activities that included annual updates to policy and procedural manuals, monitoring of equipment calibrations, and recording fire safety plans and drills and in-service attendance records. Indeed, many clinic directors reported RM infractions, suggesting that students had some exposure to information regarding RM infractions, most often client documentation mistakes, and issues related to public safety, externship contracts, and privacy. In addition, some of the programs had evacuation procedures, and six had prior campuswide lockdowns.

School lockdowns are included as contemporary concerns that can impact students and professionals in the field. Alarms or announcements within a building may indicate a safety concern and/or individuals may sign up to receive immediate text messages regarding safety lockdowns, emergencies and other planned incidents within a locality. These safety alerts may be related to an issue within a school setting, university or a particular location in a neighborhood. Professionals must act to protect themselves and the clients within the classroom or clinic setting.

An additional contemporary issues that graduate students should be exposed to is how to handle suspected abuse/bullying cases. Professionals are mandated reporters and should be aware of the state laws where they practice (Johnson, 2012). This is an issue that has been addressed in various publications by ASHA, as bullying of children with communication disorders, hearing loss and disabilities has been noted (McKinley, 2004; Blood, 2014; Hughes, 2014). Evidence of exposure to and retention of information related to the safety and welfare of the patients is certainly warranted considering the issues that professionals may face, and state licensure may require that professionals working in schools participate in harassment, bullying, abuse and discrimination prevention, and intervention trainings. Graduate students and professionals should be encouraged to obtain information regarding the requirements of the state in which they practice. 
Further, Mashima \& Doarn (2008), discuss the expanding role of SLPs in the area of telehealth, and discuss potential legal issues related to evidence-based practice, clinical competency, informed consent and providing education and training to assistants and/or caregivers. With the expanding scope of practice, graduate students and professionals need to ensure they are safeguarding patients and themselves by being aware of potential risks.

A particular issue that can arise for clinical training programs is the existence of students who can perform satisfactorily in academic courses, yet lack the skills deemed necessary to move forward with clinical internships/externships. Programs need to have protocols in place to deal with students who lack clinical competence. The importance of consistently following protocols and detailed documentation is stressed throughout the RM literature for various clinical training programs (Gilfoyle, 2008; Johnstone \& Kanitsaki (2008); Kolbler and Lucado, 2005; Vincent et al, 2005). Gilfoyle discusses the potential legal ramifications of clinic programs not addressing risks to students, faculty and the public, and provides guidance on how programs can address student competency issues in the clinical setting. The importance of detailed documentation of instances of concern, written notices being provided to students and obtaining students' signatures on remediation plans are considered by Gilfoyle (2008, p. 203) to be "critical risk management tools." In addition, the importance of updating academic and clinical policies, ensuring that faculty, administrators and students are aware of the policies and that such policies are fair and consistently enforced is stressed.

Hopkin (2017) suggests individuals and organizations should develop RM programs that are "proportionate, aligned, comprehensive, embedded and dynamic (PACED)" (p. 441). Proportionate refers to identifying the level of risks that may arise in the particular environment. The risks should align with the culture of the organization and the RM should be embedded throughout the various manuals and protocols of the facility. In addition, the RM procedures should comprehensively cover all of the risks the individual and/or organization may encounter and be dynamic and responsive to the changing needs of the organization and employees. In the case of clinical programs, protocols should be analyzed and updated, as needed, based on where applicable, the laws, accreditation guidelines, evidence based practice, incidents in clinic, etc.

The results obtained from this survey suggest that pre-professional candidates have some, but not consistent and comprehensive exposure to RM content and/or activities. From a survey tool alone, it is difficult to identify the degree to which university clinics address the wide range of RM topics; however, as derived from these data, it appears that pre-professional RM training is only somewhat available and narrow in scope. Such a finding is inconsistent with CFCC and CAA standards and thus a gap may exist between policy and practice.

Weaknesses. There are several weaknesses that limit the generalization of these findings. First, despite extensive survey development, this new RM survey tool has not yet been proven as valid and reliable. Future RM work will allow for continued development of an effective survey tool. Second, although the survey link was emailed to university clinic directors whose email addresses were obtained from a 280-member CAPCSD database and respondents were asked to indicate how many years they worked in their "directorial role," this research did not confirm the respondents' titles, nor did it verify that the participants had sufficient knowledge of their programs' academic requirements. Future research might triangulate data collection strategies via surveys of academic 
program directors and graduating students, as well as reviewing graduate and undergraduate syllabi.

Future Directions and Research. University clinics should examine the content and scope of their RM offerings. The following questions might prove helpful as programs attempt to identify the degree to which RM is embedded within coursework and clinical experiences: 1) Is RM information included in and updated in the program and clinic manuals?; 2) Is RM information addressed during graduate school and clinic orientation meetings?; 3) How might RM be embedded into formative and summative assessment activities?; 4) Does the graduate program address a myriad of RM issues?; 5) Are faculty, administrators and students are aware of the clinic policies, and are said policies fair and consistently enforced?; and 6) Does the program have clear guidelines for dealing with student clinical competencies issues?

Future research might consider which pedagogical strategies best acculturate undergraduate and graduate students to RM issues and when might these activities best be introduced during professional development. For example, there may be differences in pedagogy for undergraduate versus graduate students, and certain topics may be better incorporated into beginning versus advanced practicum experiences. Also, it would be helpful to develop reliable and valid assessment tools for rating students' RM knowledge.

Conclusion. Although RM is a contemporary topic across many American private and publicinterest industries, this survey's results suggest that university speech-language-hearing clinics are only somewhat vested in RM topics and pedagogy. University clinics, ASHA, and AAA are encouraged to further evaluate the content and breadth of candidates' knowledge and experiences to ensure that RM coursework and resources address this contemporary and critical topic.

\section{References}

Accreditation Commission for Audiology Education (2016). Accreditation standards for the Doctor of Audiology Program. Retrieved from http://acaeaccred.org/standards/.

American Academy of Audiology (2016). Code of Ethics. Retrieved from http://www.audiology.org/publications-resources/document-library/code-ethics.

American Society for Healthcare Risk Management (n.d.). Retrieved 4/30/17 from http://www.ashrm.org/.

American Speech-Language-Hearing Association (1994). Professional liability and risk management for the audiology and speech-language pathology professions. ASHA, 36, 25-38.

American Speech-Language-Hearing Association (2007). ASHA Practice Policy. Retrieved from http://www.asha.org/policy/type/

American Speech-Language-Hearing Association (2016) Code of Ethics. Retrieved from http://www.asha.org/Code-of-Ethics/.

Bal, B. S. (2009). An introduction to medical malpractice in the United States. Clinical Orthopaedics and Related Research, 467(2), 339-347.

Blood, G. (2014). Bullying be gone: The power of responsible bystanders is tremendous, and that's where we - as role models and advocates for our often vulnerable clients - come 
in. but what, exactly, to do in cases of bullying isn't always so clear. These pointers can help. The ASHA Leader, 19(5), 36-42.

Brancato, G., Macchia, S., Murgia, M., Signore, M., Simeoni, G., Blanke, K., \& HoffmeyerZlotnik, J. (2006). Handbook of recommended practices for questionnaire development and testing in the European statistical system. European Statistical System. Retrieved from http://www.istat.it/en/files/2013/12/Handbook_questionnaire_development_2006.pdf

Council on Academic Accreditation in Audiology and Speech-Language Pathology. (2016). 2017 Standards for Accreditation. Retrieved from http://caa.asha.org/reporting/standards/2017-standards/.

Council for Clinical Certification in Audiology and Speech-Language Pathology of the American Speech-Language-Hearing Association. (2012). 2012 Standards for the Certificate of Clinical Competence in Audiology. Retrieved 1/02/2018 from http://www.asha.org/Certification/2012-Audiology-Certification-Standards/.

Dionne, G. (2013). Risk management: History, definition, and critique. Risk Management and Insurance Review, 16(2), 147-166.

Farley, D., Zheng, H., Rousi, E., \& Leotsakos, A. (2015). Field test of the world health organization multi-professional patient safety curriculum guide. PloS One, 10(9), e0138510.

Gilfoyle, N. (2008). The legal exosystem: Risk management in addressing student competence problems in professional psychology training. Training and Education in Professional Psychology, 2(4), 202.

Hopkin, P. (2017). Fundamentals of risk management: understanding, evaluating and implementing effective risk management. New York, NY: Kogan Page Publishers.

Hughes, S. (2014). Bullying: What speech-language pathologists should know. Language, Speech, and Hearing Services in Schools, 45(1), 3-13.

The Joint Commission (2016). The Joint Commission: Over a century of quality and safety. Retrieved from https://www.jointcommission.org/assets/1/6/TJC_history_timeline_through_2015.pdf.

Johnson, H. (2012). Protecting the most vulnerable from abuse. The ASHA Leader, 17(14), 1619.

Johnstone, M., Kanitsaki, O. (2007). Clinical risk management and patient safety education for nurses: A critique. Nurse Education Today. Volume 27, Issue 3, 185-191.

Johnstone, M., Kanitsaki, O. (2008). Patient safety and the integration of graduate nurses into effective organizational clinical risk management systems and processes: An Australian study. Quality Management in Healthcare, 17(2), 162-173.

Kolber, M., \& Lucado, A. M. (2005). Risk management strategies in physical therapy: Documentation to avoid malpractice. International Journal of Health Care Quality Assurance, 18(2), 123-130.

Leggat, S.G., Phillips, B., Pearce, P., Dawson, M., Schulz, D., \& Smith, J. (2016) Clinical supervision for allied health staff: Necessary but not sufficient. Australian Health Review, 40, 431-437. 
Lubinski, R., \& Hudson, M. W. (2013). Professional Issues in Speech-Language Pathology and Audiology ( $4^{\text {th }} e d$.). Clifton Park, NY: Cengage Learning.

Mashima, P. A., \& Doarn, C. R. (2008). Overview of telehealth activities in speech-language pathology. Telemedicine and e-Health, 14(10), 1101-1117.

McKinley, N. (2004). Braving the bullies: What speech-language pathologists can do. The ASHA Leader Online, 16, 17.

Qualtrics (2014). Qualtrics software (Version: November 2014). Provo, UT: Qualtrics. Retrieved from http://www.qualtrics.com

Streimelweger, B., Wac, K., \& Seiringer, W. (2015). Improving patient safety through humanfactor-based risk management. Procedia Computer Science, 64, 79-86.

Vincent, C., Taylor-Adams, S., Chapman, E. J., Hewett, D., Prior, S., Strange, P., \& Tizzard, A. (2000). How to investigate and analyse clinical incidents: Clinical risk unit and association of litigation and risk management protocol. BMJ: British Medical Journal, 320(7237), 777.

World Health Organization (2011). Patient Safety Curriculum Guide: Multi-Professional Issue. Retrieved 01/02/18 from http://www.who.int/patientsafety/education/mp_curriculum_guide/en/ 


\section{Appendix}

Survey questions were related to the RM practices within university CSD programs. Unless otherwise specified, the choices were yes/no/not sure.

1. Does your university administration run an institution-wide environmental health and safety department (risk management program)?

2. Does university administration include your clinic or CSD program as a member of its institution-wide environmental health and safety program (risk management team)?

3. In your clinic, do you have a Risk Management Committee?

4. What are the barriers to having a clinic risk management committee (please select all that apply)?

- A Risk Management Committee has never been considered

- Lack of time

- Insufficient resources to pay for a risk management committee and its programs

- Lack of personnel to add this task to their work load

- Lack of knowledge about risk management

- My institution's administration includes our department in their overall management activities

- The need for a risk management committee is unclear

- Other (Please specify)

5. At your clinic, who is on your Risk Management Committee (please select all that apply)?

- Clinic director

- Assistant clinic director

- CSD academic faculty

- Clinical educator(s)

- Clerical staff

- Business manager

- Associate dean or other college administrator

- Undergraduate student(s)

- Graduate student(s)

- Client(s)

- Risk management consultant from outside of college/university

- Representative from your institution's environmental health and safety department (risk management)

- Other (Please specify)

6. What areas of risk management does the clinic committee consider (please select all that apply)?

- Abuse issues (e.g., elder, emotional, physical, sexual) 
- Asthma and respiratory allergies

- Chemicals (e.g., cleaning solutions)

- Client/patient documentation (e.g., charts, notes, raw data, etc.)

- Compliance to Privacy Policy

- Credentialing documentation (e.g., license, certification, liability insurance, etc.)

- Equipment calibration

- Externship contracts

- Fire safety plans

- Food allergies

- Infection control protocol

- Mobility transfer for patients (e.g., walker to stationary chair, wheelchair to washroom, etc.)

- Patient satisfaction

- Patient privacy (HIPAA)

- Public safety plan (emergency preparedness)

- Other (Please specify)

7. How frequently does your clinic Risk Management Committee meet?

- Weekly

- Monthly

- Bi-monthly

- Once a semester

- Annually

- Bi-annually

8. How is your clinic Risk Management Committee funded (please select all that apply)?

- Risk Management Committee receives no funding

- Department budget

- Clinic budget

- University budget

- Other (Please specify)

9. How long have you had a risk management program in your clinic?

- Less than 6 months

- Less than one year

- 1-2 years

- 3-5 years

- $6+$ years

10. What metric surveys are conducted by your clinic's Risk Management Committee (please select all that apply)?

- None

- Chart audits 
- Patient satisfaction survey

- Credentialing review (e.g., valid insurance, license renewal, etc.

- Externship contract validity

- Equipment calibration (e.g., biologic monitoring, electro-acoustic calibrations, etc.)

- Monitoring of cleaning (e.g., toys, ear probes, counter tops, etc.)

- Chemical expiration date monitors (e.g., ultrasonic cleaning solutions, ear mold materials, etc.)

- Fire drills (frequency and attendance)

- Safety drills (frequency and attendance)

- I do not know

- Other (Please specify)

11. Whether or not you have a clinic Risk Management Committee, which of these incidents have you recorded over the past 12 months (please select all that apply)?

- None; we did not record any risk management incidents (e.g., elder, emotional, physical, sexual)

- Asthma and/or respiratory allergy problems

- Food allergies (e.g., reaction to food exposure)

- Infection control (e.g., not cleaning materials, reusing speculae, not using gloves, etc.)

- Chemical (e.g., expiration, spills, etc.)

- Client/patient chart documentation (e.g., missing data, misfiled charts, breach of privacy)

- Credentialing documentation (e.g., expired license, certification, liability insurance)

- Externship contracts issues (e.g., sending student to site with expired or no contract)

- Fire safety (e.g., any type of fire incident)

- Lockdown, university wide

- Lockdown, in clinic only

- Patient satisfaction (e.g., unresolved complaint)

- Public safety (e.g., falls in clinic, assault, threats, etc.)

- Privacy (e.g., breach of HIPAA and privacy rules)

- Other (Please specify)

12. Regarding electronic health records, which of the following is applicable to your clinic?

- We do not maintain electronic health records at this time and have no plans to move towards electronic health records within the next 12 months.

- We do not maintain electronic health records at this time but we do plan to move towards electronic health records within the next 12 months

- We currently maintain electronic health records.

13. What is your perception regarding the use of electronic health records in your clinic?

- Documentation risks have decreased.

- Additional privacy and documentation risks have resulted. 
- Documentation and privacy risks remain unaffected.

14. How many HIPAA/Privacy incidents* have you observed and/or recorded over the past 12 months (click and drag/slide the cursor for a number, or click "Not Applicable")? An "incident" may be defined as any breach of confidentiality whether verbal (e.g., discussing clients in a public area), written (e.g., leaving charts unfiled, printing reports with identifiable information, etc.) or electronic (e.g., entering a client's name or other identifiable information in an email, leaving a computer with client information unsecured or unattended, etc.)

15. Indicate those who receive HIPAA/Privacy training and if the training is formal or informal? For those who do not receive training, select "not applicable"

\begin{tabular}{|l|l|l|l|}
\hline & Formal & Informal & Not Applicable \\
\hline Clinic director & & & \\
\hline Assistant clinic director & & & \\
\hline Clinical educators & & & \\
\hline Clerical staff & & & \\
\hline Business manager & & & \\
\hline $\begin{array}{l}\text { Associate dean or other } \\
\text { college/university administrator }\end{array}$ & & & \\
\hline Undergraduate students & & & \\
\hline Graduate students & & & \\
\hline Clients & & & \\
\hline $\begin{array}{l}\text { Risk management consultant from } \\
\text { outside of college/university }\end{array}$ & & \\
\hline $\begin{array}{l}\text { Representative from your institution's } \\
\text { environmental health and safety } \\
\text { department (risk management) }\end{array}$ & & & \\
\hline Other & & & \\
\hline
\end{tabular}

16. Identify educational activities within your risk management program

- We offer no activities

- Approved Continuing Education (CE) events

- In-service programs

- Risk Management Day (i.e., a designated time of activities dedicated to risk management awareness)

- Fire drills

- Safety/evacuation drills

- Educational brochures

- Signage 
- Webpage information

- Other (Please specify)

17. Whether or not there is a committee, what monitoring activities are conducted in your clinic and how often?

\begin{tabular}{|l|l|l|l|l|}
\hline & Never & $\begin{array}{c}\text { Once a } \\
\text { semester }\end{array}$ & Once a year & $\begin{array}{c}\text { Every other } \\
\text { year }\end{array}$ \\
\hline Document audits & & & & \\
\hline Equipment audits & & & & \\
\hline $\begin{array}{l}\text { Chemical and Material Safety } \\
\text { Data Sheet (MSDS) reviews }\end{array}$ & & & & \\
\hline $\begin{array}{l}\text { Policy and procedure manual } \\
\text { updates }\end{array}$ & & & & \\
\hline Fire drill attendance & & & & \\
\hline Safety drill attendance & & & & \\
\hline
\end{tabular}

18. Has your clinic Risk Management Program resulted in any of the following (please select the most appropriate answer below):

- We do not have a risk management program.

- Our legal department and/or administration has utilized clinic's documentation.

- We had one or more incident(s) for which we were appropriately prepared.

- We have not seen a positive or negative impact from having a risk management program.

- We had one or more incident(s) for which we were unprepared

19. Is risk management used as a clinical/academic teaching tool (please select all that apply)?

- Risk management is not embedded in any form in our curriculum.

- Risk management is embedded in at least one course in our curriculum.

- We discuss risk management subjects during student meetings outside regular classes.

- We require students to attend risk management program activities.

- Students have the option to attend risk management program activities.

- Other (Please specify)

20. How do you confirm your students' knowledge of risk management issues?

- Students' knowledge is not confirmed via assessment tool.

- Students' knowledge is confirmed via assessment tool.

- Students perform self-evaluation of risk management knowledge.

- Other (Please specify)

21. Please select the state in which your clinic is located. 
22. What services are offered in your college/university clinic (please select all that apply)?

We do not have a university clinic

Audiology

Speech-Language Pathology

Dentistry

General Medicine

Learning Disabilities/Special Education

Reading

Nursing

Occupational Therapy

Physical Therapy

Psychology

Social Work

Other

23. What is the average number of client visits per week to your clinic in one of your typical semesters (click and drag/slide the cursor for a number, or click Not Applicable Number of Speech-Language, Voice diagnostic evaluations performed on average, each week Number of SLP treatments/therapy given in your clinic on average, each week Audiological diagnostic services given in your clinic on average, each week (combine CAE, APD, ABR, ENG, etc.) Hearing aid services of any type given in your clinic, on average, each week (includes HAE, Real Ear, counseling, earmold, ALDs, etc.)

Hearing screenings sponsored and performed on average, each week by your clinic (combine those off-site and on campus) Other

24 . What is the average number of personnel entering your clinic on weekly basis in one of your typical semesters (click and drag/slide the cursor for a number, or click Not Applicable)?

How many clinical educators/supervisors work in your clinic each week? How many faculty, full time and adjunct, come into your clinic each week to work, teach, consult or do research, on average?

How many work study students/TAs work in your clinic each week? How many clerical support personnel work in your clinic each week? Other

25. What is your student clinician population? Undergraduates Graduate SLP Graduate AuD

26. On average, how many students enter your clinic each week to observe versus work with client as part of their training (click and drag/slide the cursor for a number, or click Not Applicable)? Undergraduate students observing Undergraduate students working with clients Graduate SLP students observing Graduate SLP students working with clients 
Teaching and Learning in Communication Sciences \& Disorders, Vol. 2 [2018], Iss. 2, Art. 3

Graduate AuD students observing

Graduate AuD students working with clients

27. How many years have you worked in your directorial role(s)?

28. How many years have you been working in the field of communication sciences and disorders?

29. What is your area of license/certification?

Audiology

Speech-Language Pathology

Both 